\title{
Study of Generalized Hourglass Section in Carbon Nanocone via Connection Number
}

\author{
Muhammad Mubashar (D), ${ }^{1}$ Muhammad Hussain (D), ${ }^{1}$ Fatimah Abdulrahman Alrawajeh (D), \\ and Sultan Almotairi iD ${ }^{3}$ \\ ${ }^{1}$ Department of Mathematics, COMSATS University Islamabad, Lahore Campus, Islamabad 54000, Pakistan \\ ${ }^{2}$ Department of Mathematics, Faculty of Science College, Imam Abdulrahman Bin Faisal University (IAU), \\ Dammam 31441, Saudi Arabia \\ ${ }^{3}$ Department of Natural and Applied Sciences, Faculty of Community College, Majmaah University, \\ Majmaah 11952, Saudi Arabia \\ Correspondence should be addressed to Fatimah Abdulrahman Alrawajeh; falrawjih@iau.edu.sa
}

Received 3 July 2021; Accepted 25 July 2021; Published 3 August 2021

Academic Editor: Muhammad Imran

Copyright (C) 2021 Muhammad Mubashar et al. This is an open access article distributed under the Creative Commons Attribution License, which permits unrestricted use, distribution, and reproduction in any medium, provided the original work is properly cited.

In 1972, Gutman and Trinajstic showed that total $\pi$-energy of a molecule depends upon a numeric quantity which is often called as Zagreb index. In the same report, they also discussed another numeric quantity depending on the number of atoms at a distance two from a particular atom and proved influencing results on $\pi$-energy of a molecule. In modern literature, this quantity is named as connection number. In this article, we will describe some Zagreb connection numbers for hourglass section in carbon nanocone network with different lengths of cycle in the central core.

\section{Introduction}

In the theoretical branch of chemistry, molecular quantities are important for modeling structural information of different molecules. The purpose for getting this information is to capture physical and chemical properties of the molecules. To attain this, a chemical structure of a molecule is presented as graphs, and the molecular quantities obtained from a particular chemical structure are graph invariants under graph isomorphism. In the mathematical branch of chemistry, this study is termed as quantitative structural activity relationship (QSAR). Predicting electron energy and melting (boiling) point for a structure are primary applications of these studies [1].

In discrete branch of mathematics, studying mathematical structures by modeling pairwise relations between sets of objects results in graphs. Simply, a graph consists of some vertices, which are joined by edges. Commonly, the set of vertices in a graph $G$ is described by $V$, whereas the set of edges is represented by $E$. Chemical graph theory is a branch of mathematics which combines graph theory and chemistry. Graph theory is used to mathematically model molecules in order to gain insight into the physical properties of these chemical compounds. In this branch, a chemical structure for a compound is visualized as a unique form of a graph by showing its atoms as vertices and bonds between atoms as edges. This is a simple transformation of a structure into a graph which helps chemists to study physical and chemical structural properties for a particular network [2]. Very recently, a new two-dimensional bilayered naturally existing network of germanium phosphide is topologically explained in [3]. Some degree-based topological indices for p-type benzene ring for a two-dimensional network are given in [4]. A computer paradigm cellular neural network is explained by a new kind of dominating topological invariants by Ejaz et al. [5].

In 1972, Ivan Gutman and Nenad Trinajstic [6] very firstly defined a numeric quantity while studying the electron energy of a molecule. Later on, this quantity will have been called as the Zagreb index. It depends on the number of 
bonds (edges) connected to an atom. Particularly, the number of bonds attached to an atom is called degree, and Zagreb index is purely based on degrees of all atoms in a structure. After this, these same mathematicians defined another quantity while studying molecular branching of orbitals which is called the second Zagreb index. With the passage of time, the abovementioned quantities would be termed as the first and second Zagreb indices by many graph theorists and chemists. More than hundreds of researchers did work on it and published their work in highly reputed journals [7].

In $[1,8]$, these two quantities are modified and redefined as noval quantities on the basis of connection numbers, and this experiment shows very effective results in different aspects. As we know that the number of edges (bonds) is the degree of an atom (vertex), similarly, the second degree is the number of edges from a particular vertex to a vertex at a distance two. In other words, for a vertex $u$ in a graph, the number of vertices at a distance two is called the connection number for $u$ and represented as $\tau_{u}$. In the following, we will define these modified versions of Zagreb indices and are termed as the first Zagreb connection number $\left(Z_{1}\right)$, second Zagreb connection number $\left(\mathrm{ZC}_{2}\right)$, and more modified type of first Zagreb as first modified connection number $\left(\mathrm{ZC}_{1}^{*}\right)$.

$$
\begin{aligned}
& \mathrm{ZC}_{1}=\sum_{u \in V} \tau_{u}^{2}, \\
& \mathrm{ZC}_{2}=\sum_{u v \in E}\left(\tau_{u} \cdot \tau_{v}\right), \\
& \mathrm{ZC}_{1}^{*}=\sum_{u v \in E}\left(\tau_{u}+\tau_{v}\right) .
\end{aligned}
$$

\section{Carbon Nanocone}

Round about in 1968, the first appearance of a carbon nanocone comes under observation by naturally existing graphite surface. The attraction of these structures is its applications in biosensors, energy and gas storage, nanoelectric devices, and many more. These nanocones are carbon-consisting networks which are infinite undirected planar structures in theory of graphs [9].

Carbon nanomaterials have drawn focusable attention and attraction during the last two decades because of its effective physical applications in nanotechnology as emerging materials of splendid practical application. But carbon nanocones (CNCs) have drawn full attention after the discovery of free-standing structures or canonical topology as cap on one end of nanotubes (CNTs) [10-12]. CNCs are admired as alternatives of (CNTs) because of the absence of potentially poisonous metal catalyst in synthesis and mass production at room temperature [13]. In general, during the declamation of CNTs, strong acids are used in order to close out metal catalysts. In this process, deficiency introduced with the hindrance of destructing the graphite structure. On the other hand, the useful applications and attractive properties of CNCs are easy to approach. The application of CNCs as drug delivery capsules [14] and gas storage devices increased their significance in modern era. Throughout the years, this subject has been developing unique scientific obsession with planar, curved, and wrapped nanoscale structures, such as graphene, fullerenes, and nanotubes [15]. It has a strong technological interest just as their innovative structural, noticeable electronic and mechanical properties. Curved carbon structures are used to investigate growth and nucleation. Especially, pentagon presence in CNCs plays vital role [16]. The 60 declination defect was detected when the pentagon inserted in a graphite sheet. This is the key of CNCs formation with pentagon as tip apex which leads us to the existence of nanotubes with tip topology [17]. This type of defects in graphite networks were theoretically considered for the study of electronic states [18]. CNCs have free-standing structures with sharp edges as these properties have applications in technology and electronics $[19,20]$. The basic cell unit of carbon nanoconeconsisting benzene ring is shown in Figure 1(a), and two benzene rings having a common edge in naphthalene is shown in Figure 1(b).

A graphical representation of these cones shows conical shape with a cycle of specific length at its core with hexagonal layers around the core. We are interested particularly in a structure with some different structural forms with $n$ layers of hexagons around the centre. In Section 3, we will explain the noval structural form derived from CNC very similar to the bow-tie shape. Javaid et al. discussed some important results related to rhombus-type silicate and oxide networks and calculated indices to study the chemical behaviour [21]. Chemical properties and chemical bonding networking in the neural network were studied significantly by using indices $[22,23]$. These gave a wider range to study the connection numbers and other indices on different chemical networks for observing the chemical behaviour and connectivity among the chemical bonds. Calculation of Zagreb connection numbers is based upon the specific distance. Formulas for many topological indices look like similar to one another. Degree-based, distance-based, and specific-restricted domain topological indices can differentiate among all of them. Leap Zagreb indices, dominating topological indies, and zagreb connection indices are close to each other by formulas but different by chemical structural properties of chemical structural-based compound [24].

\section{Hourglass Section in Carbon Nanocone}

Carbon nanocones have nice and interesting geometrical properties. These structures are connected, with infinite chains of concatenated hexagons having the property of two adjacent connected hexagons with a common edge. The construction property defines a definite rule for carbon nanostructure and it provides building block structure. That is why, it gives a planar simple connected graph obtained by connected regular hexagons [25].

Observing this geometrical property of the CNC here, we will describe its structure as similar to the hourglass. In this system, the core is centred with a cycle of specific length, whereas the layers of hexagons are around it. Moreover, this structure is similar to the doubled cone having a common 


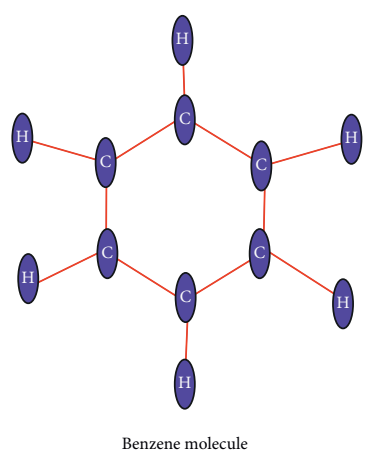

(a)

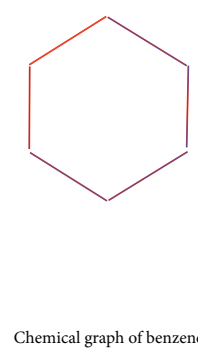

Chemical graph of benzen

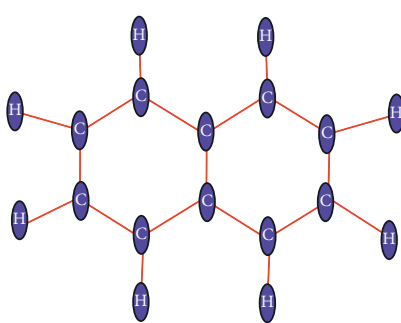

Naphthalene molecule

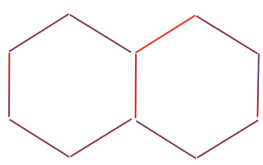

Naphthalene chemical graph

(b)

FIGURE 1: Some hydrocarbons with associated chemical graphs. (a) Benzene with chemical graph. (b) Naphthalene with chemical graph.

cycle core at the centre. We will term this unique noval system as "hourglass system carbon nanocone" (HGSCNC), whereas we will express the $n$-dimensional system as $\operatorname{HGSCNC}(n, k)$, where $n$ is the dimension of the system which is the number of hexagon layers around the core and $k$ represent the length of cycle at the core. We will discuss two cases for $k$ : (1) when $k$ is odd and (2) when $k$ is even.

3.1. When Length of Cycle $m$ Is Odd in n-Dimensional HGSCNC. In this case, we are presenting the HGSCNC with a cycle of odd length $m$ up to $n$-dimension. We will observe sharpness and scattered pattern in $n$-dimensional hexagonal layers with odd length cycle. By observing the structural property of this particular case, we have found that this structure has $2 n^{2}+12 n+m$ total number of vertices with $n \geq 2$ and $m \geq 7$, whereas the edge set has total $3 n^{2}+15 n+m$ edges with $n \geq 2$ and $m \geq 9$. Here, $n$ represents the number of hexagon layers and $m \in O$ is the length of the cycle. A simple connected graph for this particular case is shown in Figure 2 and mathematically expressed as $\operatorname{HGSCNC}(n, m)$. A graphical display for HGSCNC for $n=4$ with cycle of odd length for $m=7$ is shown in Figure 3 .

By working on some structural properties and behaviour of $\operatorname{HGSCNC}(n, m)$ upto $n$ dimensions, we found it very interesting and rapid growing network. Here, we will state a lemma about its structural behaviour given as follows.
Lemma 1. For $n \geq 2$ and $m=7$ HGSCNC, we have the first Zagreb connection number $Z C_{1}$ which is as follows:

$$
\mathrm{ZC}_{1}(\operatorname{HGSCNC}(n, 7))=72 n^{2}+192 n+58 \text {. }
$$

Lemma 2. For $n \geq 2$ and $m=9$ HGSCNC, we have first Zagreb connection number $Z C_{1}$, second Zagreb connection number $Z C_{2}$, and first modified Zagreb connection number $Z C_{1}^{*}$ which are as follows:

$$
\begin{aligned}
& \operatorname{ZC}_{2}(\operatorname{HGSCNC}(n, 9))=108 n^{2}+228 n+84 \\
& \mathrm{ZC}_{1}^{*}(\operatorname{HGSCNC}(n, 9))=36 n^{2}+120 n+46
\end{aligned}
$$

3.1.1. Main Results. In this section, we will state our main results about connection numbers with the help of the abovementioned lemma and its consequences. These results are related to Zagreb connection numbers $\operatorname{HGSCNC}(n, m)$.

Theorem 1. Hourglass carbon nanocone system holds closed expression for first Zagreb connection number $Z C_{1}$, second Zagreb connection number $Z C_{2}$, and first modified Zagreb connection number $Z C_{1}^{*}$ which are as follows:

$$
\begin{aligned}
& Z_{1}(\operatorname{HGSCNC}(n, m))=72 n^{2}+192 n+4 m+30 \forall n \geq 2 \text { and } m \geq 7, \\
& \operatorname{ZC}_{2}(\operatorname{HGSCNC}(n, m))=108 n^{2}+228 n+4 m+40 \forall n \geq 2 \text { and } m \geq 9, \\
& Z_{1}^{*}(\operatorname{HGSCNC}(n, m))=36 n^{2}+120 n+4 m+70 \forall n \geq 2 \text { and } m \geq 9 .
\end{aligned}
$$

Proof. Working on Lemma 1, we have found some properties of the vertex set in Table 1 and edge set properties in Table 2 concluded as a result of 2 for $\operatorname{HGSCNC}(n, m)$ depending on the connection number of carbon atoms in the $n$-dimensional network. By using equation (1), we have

$$
\begin{gathered}
\operatorname{ZC}_{1}(\operatorname{HGSCNC}(n, m))=4(m-3)+9(10)+16(12 n-10)+25(2)+36\left(2 n^{2}-1\right)+49(2) \\
\Rightarrow \operatorname{ZC}_{1}(\operatorname{HGSCNC}(n, m))=72 n^{2}+192 n+4 m+30
\end{gathered}
$$




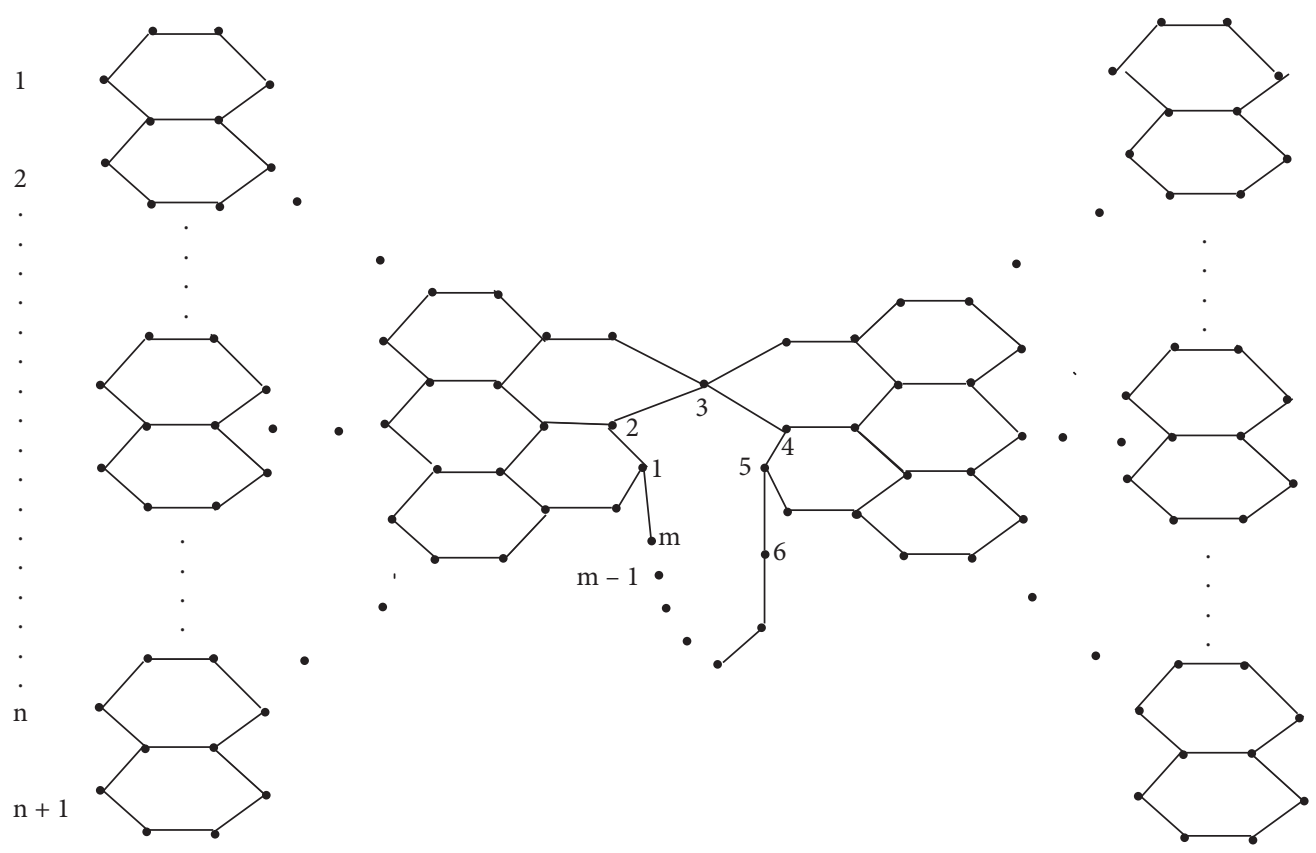

FIGURE 2: Generalized hourglass carbon nanocone $\operatorname{HGSCNC}(n, m)$.

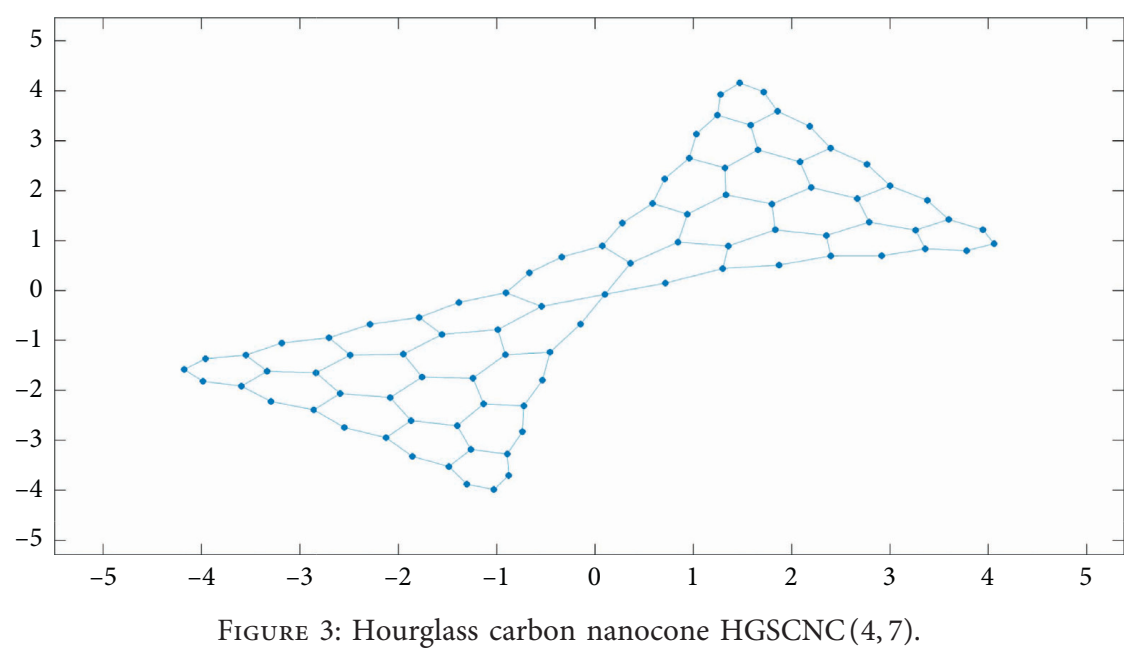

TABle 1: Vertex partition of $\operatorname{HGSCNC}(n, m) \forall n \geq 2$ and $m \geq 7$ with respect to connection number $\tau$.

\begin{tabular}{lcccccc}
\hline$\tau_{u}$ & 2 & 3 & 4 & 5 & 6 & 7 \\
\hline Number of vertices & $m-3$ & 10 & $12 n-10$ & 2 & $2 n^{2}-1$ & 2 \\
\hline
\end{tabular}

TABle 2: Edge partition of $\operatorname{HGSCNC}(n, m) \forall n \geq 2$ and $m \geq 9$.

\begin{tabular}{lcccccccccc}
\hline$\left(\tau_{u}, \tau_{v}\right)$ & $(2,3)$ & $(4,3)$ & $(4,4)$ & $(4,5)$ & $(6,6)$ & $(4,6)$ & $(5,6)$ & $(6,7)$ & $(4,7)$ & $(2,2)$ \\
\hline Number of edges & 10 & 10 & $12 n-16$ & 2 & $3 n^{2}-3 n-2$ & $6 n-4$ & 2 & 4 & 2 & $m-8$ \\
\hline
\end{tabular}


From equation (2) and by using the abovementioned connection numbers for different edges, we have

$$
\begin{aligned}
\mathrm{ZC}_{2}(\operatorname{HGSCNC}(n, m))= & 6(10)+12(10)+16(12 n-16)+20(2) \\
& +36\left(3 n^{2}-3 n-2\right)+24(6 n-4)+30(2)+42(4)+28(2)+4(m-8) \\
\Rightarrow & \mathrm{ZC}_{2}\left(\mathrm{CNC}_{5}(n)\right)=108 n^{2}+228 n+4 m+40 .
\end{aligned}
$$

From equation (3) and by using the abovementioned connection numbers for different edges, we have

$$
\begin{aligned}
\mathrm{ZC}_{1}^{*}(\operatorname{HGSCNC}(n, m))= & 5(10)+7(10)+8(12 n-16)+9(2) \\
& +12\left(3 n^{2}-3 n-2\right)+10(6 n-4)+11(2)+13(4)+11(2)+4(m-8) \\
\Rightarrow & \mathrm{ZC}_{1}^{*}(\operatorname{HGSCNC}(n, m))=36 n^{2}+120 n+4 m+70 .
\end{aligned}
$$

3.2. When the Length of Cycle $p$ Is Even in n-Dimensional $H G S C N C$. In this case, we are presenting the HGSCNC with even length $p \in E$ cycle up to $n$ dimensions. We will observe sharpness and scattered pattern in $n$-dimensional hexagonal layers with even length cycle. On observing the structural property of this particular case, we have found that this structure has $2 n^{2}+8 n+p+8$ total number of vertices with $n \geq 2$ and $p \geq 10$, whereas the edge set has total $3 n^{2}+15 n+p$ edges with $n \geq 2$ and $p \geq 14$. Here, $n$ is representing the number of hexagon layers and $p \in E$ is the length of cycle. A simple connected graph for this particular case is shown in Figure 4 and mathematically expressed as $\operatorname{HGSCNC}(n, p)$. A graphical display for HGSCNC for $n=3$ with a cycle of odd length for $p=10$ is shown in Figure 5.

On working some structural properties and behaviour of $\operatorname{HGSCNC}(n, m)$ up to $n$ dimensions, we found it very interesting and rapid growing network. Here, we will state some lemmas about particular cases for connection numbers when the length of the cycle is fixed.

Lemma 3. For $n \geq 2$ and $p=10$ HGSCNC, we have first Zagreb connection number $Z C_{1}$ which is as follows:

$$
\mathrm{ZC}_{1}(\operatorname{HGSCNC}(n, 10))=72 n^{2}+128 n+156
$$

Lemma 4. For $n \geq 2$ and $p=14$ HGSCNC, we have first Zagreb connection number $Z C_{1}$, second Zagreb connection number $Z C_{2}$, and first modified Zagreb connection number $Z C_{1}^{*}$ which are as follows:

$$
\begin{aligned}
& \mathrm{ZC}_{2}(\operatorname{HGSCNC}(n, 14))=108 n^{2}+228 n+32 \\
& \mathrm{ZC}_{1}^{*}(\operatorname{HGSCNC}(n, 14))=36 n^{2}+120 n+56
\end{aligned}
$$

3.2.1. Main Results. In this section, we will state our main results about connection numbers with the help of the abovementioned lemma and its consequences. These results are related to Zagreb connection numbers for $\operatorname{HGSCNC}(n, p)$.

Theorem 2. Hourglass carbon nanocone system holds closed expression for first Zagreb connection number $Z C_{1}$, second Zagreb connection number $Z C_{2}$, and first modified Zagreb connection number $Z_{1}^{*}$ which are as follows:

$$
\mathrm{ZC}_{1}(\operatorname{HGSCNC}(n, p))=72 n^{2}+128 n+4 p+116
$$

$\forall n \geq 2$ and $p \geq 10$

$$
\mathrm{ZC}_{2}(\operatorname{HGSCNC}(n, p))=108 n^{2}+228 n+4 p-24
$$

$\forall n \geq 2$ and $p \geq 14$

$$
\mathrm{ZC}_{1}^{*}(\operatorname{HGSCNC}(n, p))=36 n^{2}+120 n+4 p \forall n \geq 2 \text { and } p \geq 14 \text {. }
$$

Proof. Working on the lemma, we have found some properties of the vertex set in Table 3 and edge set in Table 4 of $\operatorname{HGSCNC}(n, p)$ depending on the connection number of carbon atoms in the $n$-dimensional network. By using equation (1), we have 


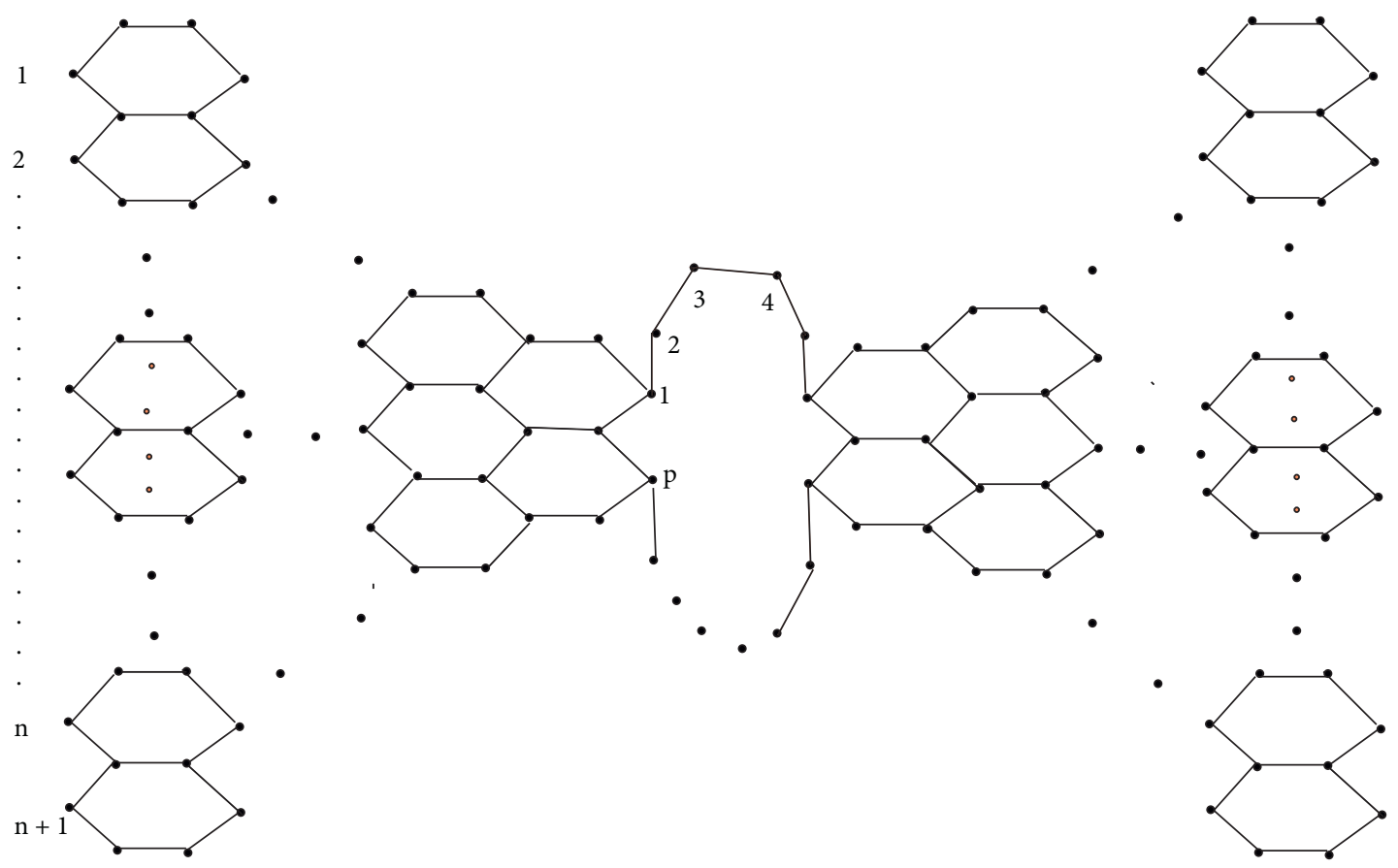

FIGURE 4: Generalized hourglass carbon nanocone $\operatorname{HGSCNC}(n, p)$.

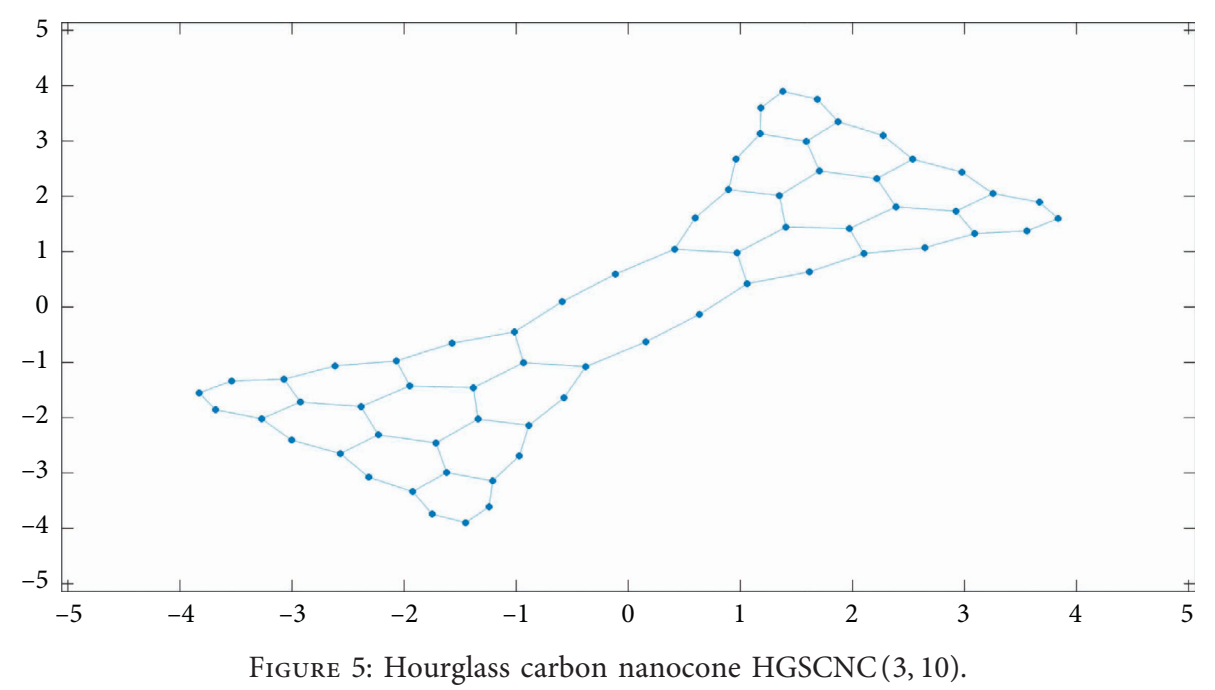

$$
\begin{aligned}
\operatorname{ZC}_{1}(\operatorname{HGSCNC}(n, p)) & =4(p-6)+9(12)+16(8 n+2)+36\left(2 n^{2}\right) \\
& \Rightarrow \mathrm{ZC}_{1}(\operatorname{HGSCNC}(n, p))=72 n^{2}+128 n+4 p+116
\end{aligned}
$$

From equation (2) and by using the abovementioned connection numbers for different edges, we have

$$
\begin{gathered}
\operatorname{ZC}_{2}(\operatorname{HGSCNC}(n, p))=6(12)+12(12)+16(12 n-12)+36\left(3 n^{2}-3 n\right)+24(6 n)+4(p-12) \\
\Rightarrow Z_{2}(\operatorname{HGSCNC}(n, p))=108 n^{2}+228 n+4 p-24
\end{gathered}
$$


Table 3: Vertex partition of $\operatorname{HGSCNC}(n, p) \forall n \geq 2$ and $p \geq 10$.

\begin{tabular}{lccccc}
\hline$\tau_{u}$ & 2 & 3 & 4 & 6 \\
\hline Number of vertices & $p-6$ & 12 & $8 n+2$ & $2 n^{2}$ \\
\hline
\end{tabular}

TABle 4: Edge partition of $\operatorname{HGSCNC}(n, m) \forall n \geq 2$ and $p \geq 14$.

\begin{tabular}{lcccccc}
\hline$\left(\tau_{u}, \tau_{v}\right)$ & $(2,3)$ & $(4,3)$ & $(4,4)$ & $(6,6)$ & $(4,6)$ & $(2,2)$ \\
\hline Number of edges & 12 & 12 & $12 n-12$ & $3 n^{2}-3 n$ & $p n$ & $p-12$ \\
\hline
\end{tabular}

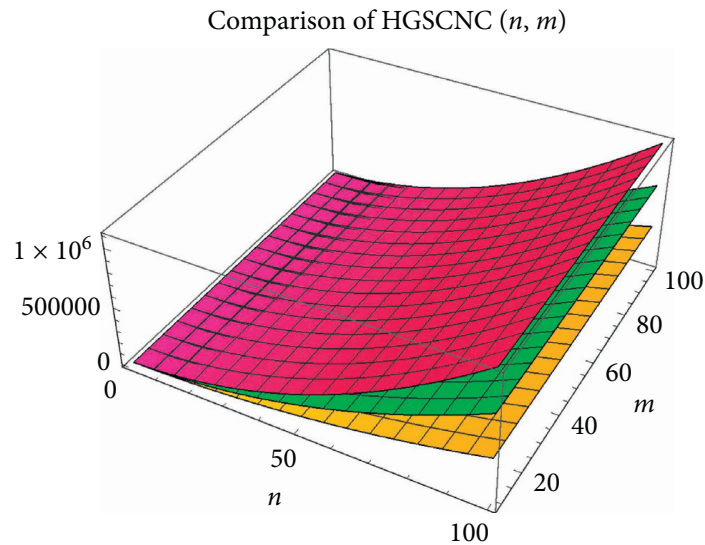

ZC1

$\square \mathrm{ZC2}$

$\mathrm{ZC1}$

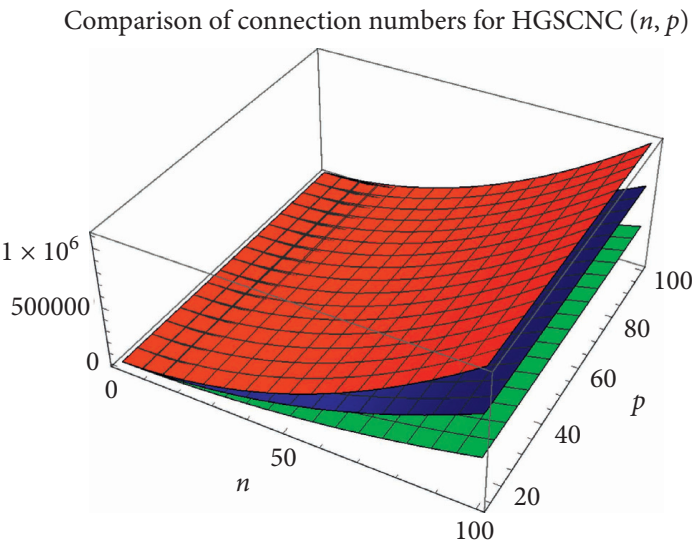

$\square \mathrm{ZC}_{1}$
$\square \mathrm{ZC}_{2}$
$\square \mathrm{ZC}_{1}$

(a)

(b)

Figure 6: Comparison of connection number for HGSCNC. (a) Comparison of $\mathrm{ZC}_{1}, \mathrm{ZC}_{2}$, and $\mathrm{ZC}_{1}^{*}$ for HGSCNC ( $n$, $m$ ). (b) Comparison of $\mathrm{ZC}_{1}, \mathrm{ZC}_{2}$, and $\mathrm{ZC}_{1}^{*}$ for $\operatorname{HGSCNC}(n, p)$.

From equation (3) and by using the abovementioned connection numbers for different edges, we have

$$
\begin{gathered}
\operatorname{ZC}_{1}^{*}(\operatorname{HGSCNC}(n, p))=5(12)+7(12)+8(12 n-12)+12\left(3 n^{2}-3 n\right)+10(6 n)+4(p-12) \\
\Rightarrow Z_{1}^{*}(\operatorname{HGSCNC}(n, p))=36 n^{2}+120 n+4 p .
\end{gathered}
$$

\section{Graphical Comparison}

In this section, we will compare our results via graphical view. Firstly, we have compared our output results among $\mathrm{ZC}_{1}, \mathrm{ZC}_{2}$, and $\mathrm{ZC}_{1}^{*}$ for $\operatorname{HGSCNC}(n, m)$, and results are shown in Figure 6(a). Similarly, we have compared our output results among $\mathrm{ZC}_{1}, \mathrm{ZC}_{2}$, and $\mathrm{ZC}_{1}^{*}$ for $\operatorname{HGSCNC}(n, p)$, and results are shown in Figure 6(b).

\section{Conclusion}

In this article, we have computed closed results for the family of Zagreb connection number in the hourglass system carbon nanocone (HGSCNC) and with its core of even length and its core of odd length. For the first time, we have evaluated the Zagreb connection numbers for $\operatorname{HGSCNC}(n, m)$ and $\operatorname{HGSCNC}(n, p)$. The results are very interesting, and we concluded that $\operatorname{HGSCNC}(n, m)$ is the fast predicting network as compared to $\operatorname{HGSCNC}(n, p)$. Furthermore, we have concluded that $\mathrm{ZC}_{2}$ has better prediction for both $\operatorname{HGSCNC}(n, m)$ as well as for $\operatorname{HGSCNC}(n, p)$. Predicting ability of a topological index encourages the theoretical study of the chemical structure. We computed Zagreb connection indices $\mathrm{ZC}_{1}, \mathrm{ZC}_{2}$, and $\mathrm{ZC}_{1}^{*}$ for HGSCNC upto $n$ dimensions for different lengths of central core. These results will facilitate the understanding of 
topological properties of the noval structure of HGSCNC. Our results will motivate to investigate new structure of different chemical structures and their line graphs and studying their topological and physical properties.

\section{Data Availability}

No data were used to support this study.

\section{Disclosure}

The paper has not been published elsewhere, and it will not be submitted anywhere else for publication.

\section{Conflicts of Interest}

The authors declare no conflicts of interest.

\section{Authors' Contributions}

All authors contributed equally to this work.

\section{Acknowledgments}

The authors extend their appreciation to the deputyship for Research and Innovation, Ministry of Education, Saudi Arabia, for funding this research work through the project number IFP-2020-17.

\section{References}

[1] R. Zahid, "Zagreb connection indices for some benzenoid systems," Polycyclic Aromatic Compounds, vol. 40, pp. 1-14, 2020.

[2] L. Yan, Y. Li, S. Hayat et al., "On degree-based and frustration related topological indices of single-walled titania nanotubes," Journal of Computational and Theoretical Nanoscience, vol. 13, no. 11, pp. 9027-9032, 2016.

[3] F. Ejaz, M. Hussain, and R. Hasni, "On topological aspects of bilayer Germanium Phosphide," Journal of Mathematics and Computer Science, vol. 22, no. 4, pp. 347-362, 2021.

[4] A. Ahmad, "On the degree based topological indices of benzene ring embedded in P-type-surface in 2D network," Hacettepe Journal of Mathematics and Statistics, vol. 47, no. 1, 2018.

[5] F. Ejaz, M. Hussain, H. Almohamedh, K. M. Alhamed, R. Alabdan, and S. Almotairi, "Dominating topological analysis and Comparison of the cellular neural network," Mathematical Problems in Engineering, vol. 2021, Article ID 6613433, 9 pages, 2021.

[6] I. Gutman and N. Trinajstić, "Graph theory and molecular orbitals. Total $\varphi$-electron energy of alternant hydrocarbons," Chemical Physics Letters, vol. 17, no. 4, pp. 535-538, 1972.

[7] D. Maji and G. Ghorai, "Computing F-index, coindex and Zagreb polynomials of the kth generalized transformation graphs," Heliyon, vol. 6, no. 12, Article ID e05781, 2020.

[8] A. Ali and N. Trinajstić, "A novel/old modification of the first Zagreb index," Molecular Informatics, vol. 37, no. 6-7, Article ID 1800008, 2018.

[9] N. Tagmatarchis, Advances in Carbon Nanomaterials: Science and Applications, CRC Press, Baco Raton, FL, USA, 2012.
[10] T. Ichihashi and Y. Ando, "Pentagons, heptagons and negative curvature in graphite microtubule growth," Nature, vol. 356, pp. 776-778, 1992.

[11] S. Iijima, P. M. Ajayan, and T. Ichihashi, "Growth model for carbon nanotubes,” Physical Review Letters, vol. 69, no. 21, pp. 3100-3103, 1992.

[12] S. Iijima, "Helical microtubules of graphitic carbon," Nature, vol. 354, no. 6348, pp. 56-58, 1991.

[13] M. Nadolska, M. Prześniak-Welenc, M. Łapiński, and K. Sadowska, "Synthesis of phosphonated carbon nanotubes: new insight into carbon nanotubes functionalization," $\mathrm{Ma}$ terials, vol. 14, no. 11, p. 2726, 2021.

[14] B. A. Ardeshana, U. B. Jani, A. M. Patel, and A. Y. Joshi, "Characterizing the vibration behavior of double walled carbon nano cones for sensing applications," Materials Technology, vol. 33, no. 7, pp. 451-466, 2018.

[15] M. Ge and K. Sattler, "Observation of fullerene cones," Chemical Physics Letters, vol. 220, no. 3-5, pp. 192-196, 1994.

[16] B. Bumbacila, O. Ori, F. Cataldo, and M. V. Putz, "Topological modeling of carbon nano-lattices," Current Organic Chemistry, vol. 21, no. 27, pp. 2705-2718, 2017.

[17] V. R. Kulli, "General reduced second Zagreb index of certain networks," International Journal of Current Research in Life Sciences, vol. 7, no. 11, pp. 2827-2833, 2018.

[18] Z. Hussain, M. Munir, A. Ahmad, and M. Chaudhary, J. Alam Khan and I. Ahmed, "Metric basis and metric dimension of 1pentagonal carbon nanocone networks," Scientific Reports, vol. 10, no. 1, p. 19687, 2020.

[19] M. Asif, M. Hussain, H. Almohamedh et al., "Study of carbon nanocones via connection Zagreb indices," Mathematical Problems in Engineering, vol. 2021, Article ID 5539904, 13 pages, 2021.

[20] W. Gao and M. R. Farahani, "Computing the reverse eccentric connectivity index for certain family of nanocone and fullerene structures," Journal of Nanotechnology, vol. 2016, Article ID 3129561, 6 pages, 2016.

[21] M. Javaid, M. U. Rehman, and J. Cao, "Topological indices of rhombus type silicate and oxide networks," Canadian Journal of Chemistry, vol. 95, no. 2, pp. 134-143, 2017.

[22] J.-B. Liu, J. Zhao, S. Wang, M. Javaid, and J. Cao, "On the topological properties of the certain neural networks," Journal of Artificial Intelligence and Soft Computing Research, vol. 8, no. 4, pp. 257-268, 2018.

[23] M. Javaid and J. Cao, "Computing topological indices of probabilistic neural network," Neural Computing and Applications, vol. 30, no. 12, pp. 3869-3876, 2018.

[24] I. Gutman, Z. Shao, Z. Li, S. Wang, and P. Wu, "Leap Zagreb indices of trees and unicyclic graphs," Communications in Combinatorics and Optimization, vol. 3, no. 2, pp. 179-194, 2018.

[25] Y. C. Kwun, A. Ali, W. Nazeer, M. A. Chaudhary, and S. M. Kang, "M-polynomials and degree-based topological indices of triangular, hourglass, and jagged-rectangle benzenoid systems," Journal of Chemistry, vol. 2018, Article ID 8213950, 8 pages, 2018. 